\title{
Mifepristone with Misorostol for Mid- Trimester Abortion - A Prospective Study
}

\author{
Dr. S.V. Nachiketha \\ Associate Professor, Department of OBG KIMS, Hubli
}

\begin{abstract}
:
Background: There is an increase in number of second trimester pregnancy termination due to better prenatal screening. Medical methods, using combination of mifepristone and Misoprostol, frequently being used for midtrimester pregnancy termination.

Objective: Objective of study was to study safety and effectiveness of combination of mifepristone and Misoprostol for mid-trimester MTP.

Material \& Methods: A prospective study of 68 women who underwent Mid-trimester MTP (between 13-20 weeks gestation) Karnataka Institute of Medical Sciences Hubli, Karnataka, India during 2012 - June 2015 Results: Data was analysed for 48 cases who fulfilled the inclusion criteria. Complete abortion occurred in $36(75 \%)$ of cases, $13(25 \%)$ required dilatation \& curettage for incomplete abortion including manual removal of placenta in 8 cases. There were seven cases with previous LSCS all of whom had complete abortion.

Conclusion: Combination on Mifepristone \& Misoprostol is an effective \& safe method for mid-trimester MTP and can be used in cases of previous caesarean section with caution.
\end{abstract}

\section{Introduction}

Termination of pregnancy before the period of viability is called abortion. Mid trimester abortion is termination of pregnancy between 13- 28 weeks gestation. Indian MTP law (Medical termination of pregnancy law, 1972) permits abortion till 20 weeks period of gestation ' by a registered medical practitioner provided a 11 the pre-requisites are met. (1) Mid-trimester abortions (13-20 weeks) constitute 10-15\% of all abortion cases but are responsible for two-thirds of all major complications \{WHO, 1997 (2)\}. Though majority of miscarriage occur during first trimester but recently there is gradual increase in number of mid-trimester abortion due to better prenatal screening modalities. Due to advancement in prenatal screening many of lethal malformation can be diagnosed before 20 weeks and are amenable to termination.(3). The medical methods, especially prostaglandins, have good success rates and less complication rates. So, medical methods have become increasingly popular for mid-trimester abortions.

Prostanglandins (PG El, PGE2 and PG F2a) has been ' used for mid-trimester pregnancy termination in the last 20 years. When prostaglandin $\mathrm{El}$ analogs (gemeprost or Misoprostol) are used alone for second trimester MTP, the mean induction-abortion interval (IAI) can be as long as 12-16 hours (4,5). Pretreatment with an antiprogesterone (mifepristone) prior to prostaglandin administration softens the cervix, increases the sensitivity to prostaglandins and thus converts the quiet pregnant uterus into an organ of spontaneous activity(6) leading to reduction in JAI, the total dose of prostaglandins required as well as the analgesia requirement $(5,7)$. The combination of mifepristone followed by misoprostol has been found safe and effective for mid trimester termination of pregnancy in various studies. $(3,8,9)$

The present study was conducted to find effectiveness of combination of Mifepristone and successive doses of vaginal misoprostol for mid-trimester abortions, to observe the course and outcome of abortion using the above protocol and to study any side effects of the above regimen.

\section{Material And Methods}

This prospective study was done in a tertiary care teaching hospital, after taking approval from ethical committee of the institute. A total of 48 women, who presented to us for termination of pregnancy between 13-20 weeks period of gestation due to various indications were included in the study in accordance with the inclusion criteria. They were explained about the procedure of medical termination of pregnancy, and a written informed consent was taken from each of them after explaining complications and success rate of procedure. These women were admitted in the labour ward and baseline investigations were done.

\section{Inclusion criteria}

All patients who were admitted in the labour ward seeking medical termination of pregnancy due to various reasons between 13-20 weeks period of gestation and fulfilling all the prerequisites of the MTP Act were included in the study. Study period was from January 2012 to June 2015. Patients with up to two previous 
uterine surgeries, including previous two cesarean section were also included in the study.

\section{Exclusion criteria}

1. Women who were haemodynamically unstable at the time of presentation.

2. Women, who came with inevitable or incomplete abortion.

3. Women with known heart disease, uncontrolled hypertension (BP > 160/100 mm Hg), bronchial asthma or coagulation disorder,

4. Women on anti-coagulant or corticosteroids.

5. Haemoglobin $<8$ gm $\%$.

6. Known hypersensitivity to mifepristone or Misoprostol.

7. Pregnancy beyond 20 weeks period of gestation.

8. Women not fulfilling the pre-requisites of Indian MTP act, 1972

9. Previously taken medical abortion treatement.

After admission, Mifepristone $200 \mathrm{mg}$ was administered per oral under supervision to these patients. Following after 48 hours, Misoprostol $400 \mathrm{mcg}$ was administered per vaginally. Thereafter Misoprostol was administered at a dose of $100-200 \mathrm{mcg}$ which was repeated at 4-6 hourly intervals depending on cervical findings, intensity and frequency of uterine contractions and history of previous uterine surgeries. Maximum dosage of Misoprostol administered was $2400 \mathrm{mcg}$ and the maximum time period taken for successful abortion was 48hours following administration of first dose of Misoprostol. Patients were allowed to be ambulatory according to their wish. Analgesics in the form of inj. Tramodol or tablet pracetamol were given to patients according to their discomfort level. sterile vulval pads were given which were examined at regular intervals to assess the amount of blood loss and for the expulsion of any products of conception. The time of expulsion of foetus and placenta was noted. Placenta was examined to confirm its totality. In cases with incomplete abortion i.e. when placenta was not expelled spontaneously, manual removal of placenta was done followed by check curettage. Routine check curettage was avoided so as to avoid unnecessary surgical intervention and its inherent complications and was done only when indicated i.e. in cases of incomplete abortions /excessive bleeding per vaginum. Need for blood transfusion due to excessive blood loss was recorded. Failure of procedure was defined as expulsion occurring after 48 hrs following first dose of Misoprostol, need for dilatation and evacuation, need for manual removal of placenta under anaesthesia.

\section{Following outcomes were measured -}

1. Rate of complete abortion - Complete expulsion of foetus and placenta with a maximum dose of misoprostol i.e. $2400 \mathrm{mcg}$ within 48 hours following first dose of Misoprostol.

2. Induction to delivery interval - Calculated from the time of first dose of misoprostol administration to complete expulsion of foetus and placenta.

3. Failure to achieve complete abortion within the intended time interval with maximum intended dose of misoprostol.

\section{Secondary outcomes studied were-}

1. Safety and acceptability of the method .

2. Side effects.

3. Complications observed with the regimen like excessive bleeding, incomplete abortion leading to need for emergency curettage, need for blood transfusion, sepsis, rupture uterus, need for hysterotomy/ hysterectomy.

4. Analgesia requirement.

5. Duration of hospital stay.

\section{Results}

A total of 68 women underwent mid trimester pregnancy termination during the study period. Out of these 12 women came with inevitable abortion, eight had self administered abortion pills without any prescription and thus were excluded from the study. Finally 48 cases were included in the study and analysed. The mean age of the women included in the study was 26.71 years with a range of $16-40$ years. The majority (42\%) of women were nullipara. Thirty-four women (70.83\%) were in late second trimester (16-20 weeks POG) and 14 patients (29.17\%) were in early second trimester (13-16 weeks POG). There were seven women (14.48\%) with previous caesarean section. (Table 1) The various indications for which the patients presented for medical termination of pregnancy are listed in Table 2. Thirty women (62.5\%) underwent MTP for congenital malformations amongst which neural tube defects was the commonest malformation seen in 23 patients $(47.72 \%)$ followed by renal malformations in seven patients (14.58\%). Six women underwent MTP for unwanted pregnancy, two of these were unmarried girls and rest four who were married were diagnosed to be pregnant in $2^{\text {nd }}$ trimester. They either 
had irregular bleeding ( 3 cases) or conceived in lactational amenorrhoea (lease). In nine cases $(18.75 \%)$ the reason for termination was intrauterine fetal demise, one woman $(2.08 \%)$ had partial molar pregnancy diagnosed on ultrasonography, $1(2.08 \%)$ had radiation exposure with CT scan in early pregnancy and $1(2.08 \%)$ was diagnosed with beta thalassemia major foetus on amniocentesis.

Mean induction-delivery time was 11.26 hours(95\% CI 8.23-14.28). (Table 3) There were 6 cases (12.5 $\%$ ) who aborted following mifepristone administration alone. Mean total dose of misoprostol was calculated to be 610.42mcg (95\%) CI 474.99-745.85). Mean number of doses of misoprostol required was found to be 2.13 (range 0-9) Mean dosage interval was found to be 5.7 hours (range 4-6 hours). The above regimen led to complete abortion (expulsion of foetus and placenta) in 35 cases $(75 \%)$. However 12 women $(25 \%)$ had incomplete abortion. Amongst these eight women (16.66\%) required manual removal of placenta followed by gentle check curettage. Emergency suction and evacuation for retained placental bits was required in five women $(10.42 \%)$ due to excessive bleeding per vaginum.

There were total seven patients $(14.58 \%)$ with previous caesarean section. Amongst these four patients had previous one caesarean and three patients had previous 2 caesarean section. In this group of patients the mean IAI was 13.2 hours (95\% CI 0.49-26.07) and mean total dose of Misoprostol required was 742.86 (95\% CI 347.3-1138.4) meg. All of these women with previous caesarean section presented atl8-20 weeks gestation. All of these patients had complete abortion and none required emergency suction and evacuation. No other major complication was seen in this group of patients. In Six cases $(12.5 \%)$ mean IAI was more than 24 hours (mean $37.5 \mathrm{hrs}$ ). It was observed that all these women with longer IAI were in late $2^{\text {nd }}$ trimester i.e. between $18-20$ weeks POG. The mean total dose of Misoprostol required in them was $1633 \mathrm{mcg}$ (95\% CI 1225.9-2040.7) Of these two patients $(33.33 \%)$ had incomplete abortion and required suction and evacuation.

There were no major complications seen. Side effects were few minor ones like fever. None had diarrhoea or uterine rupture requiring emergency hysterectomy (There were two patients who required blood transfusion but they were anaemic prior to the procedures There was no case of major obstetric haemorrhage observed during the study. (Table 4)

Majority didn't require any analgesics $(58.33 \%)$. Eighteen women required injectable analgesics for moderate discomfort, ten out of these $(55.55 \%)$ were primigravidae. Oral analgesics were sufficient for adequate pain relief in rest of the 2 patients $(4.17 \%)$. Hospital stay of the patients ranged from 2-7 days with a mean duration of stay being 3.4 days.

Table 1: Indications for MTP $(\mathrm{n}=48)$

\begin{tabular}{|l|l|l|}
\hline Parameter & Number & Percentage $(\%)$ \\
\hline Age (years) & & \\
\hline$<20$ & 2 & 4.16 \\
\hline $21-30$ & 37 & 77.08 \\
\hline $31-40$ & 9 & 18.75 \\
\hline Parity & & \\
\hline 0 & 20 & 41.66 \\
\hline $1-2$ & 26 & 54.17 \\
\hline$>3$ & 2 & 4.16 \\
\hline Previous abortions & & \\
\hline 0 & 32 & 66.66 \\
\hline $1-2$ & 15 & 31.25 \\
\hline$>3$ & 1 & 2.08 \\
\hline Married & 46 & 95.83 \\
\hline Unmarried & 2 & 4.17 \\
\hline POG at time of MTP & & \\
\hline $13-16$ weeks & 14 & 29.17 \\
\hline $17-20$ weeks & 34 & 70.83 \\
\hline PreviousLSCS LLScaesarean section & 7 & 14.58 \\
\hline
\end{tabular}

Table 2: Indications for MTP $(n=48)$

\begin{tabular}{|l|l|l|}
\hline Indication & Number & Percentage (\%) \\
\hline CMF & 30 & 62.5 \\
\hline Neural tube defects & 23 & 47.92 \\
\hline Renal malformations & 7 & 14.58 \\
\hline Intrauterine fetal demise & 9 & 18.75 \\
\hline Partial molar pregnancy & 1 & 2.08 \\
\hline Radiation exposure & 1 & 2.08 \\
\hline Beta thalassemia major & 1 & 2.08 \\
\hline Unwanted pregnancy & 6 & 12.5 \\
\hline
\end{tabular}


Table 3: Details of MTP procedure $(n=48)$

\begin{tabular}{|l|l|l|}
\hline & Mean (95\% CI) & Range/ no. (\%) \\
\hline Total dose of Misoprostol & $610.42(474.99-745.85)$ & $0-2400(\mathrm{Meg})$ \\
\hline Total number of doses & $2.13(1.56-2.73)$ & $0-9$ \\
\hline Dosing interval & 5.7 hours $(5.45-5.91)$ & $4-6$ hours \\
\hline Induction-abortion interval & $11.26(8.23-14.28)$ & $(3-40)$ \\
\hline $0-6 \mathrm{hrs}$ & & $7(14.58 \%)$ \\
\hline $7-12 \mathrm{hrs}$ & & $26(54.14 \%)$ \\
\hline $13-18 \mathrm{hrs}$ & & $2(4.17 \%)$ \\
\hline $19-24 \mathrm{hrs}$ & & $1(2.08 \%)$ \\
\hline$>24 \mathrm{hrs}$ & & $6(12.5 \%)$ \\
\hline Abortion with mifepristone alone & & $6(12.5 \%)$ \\
\hline Complete abortion & & $36(75.00 \%)$ \\
\hline Incomplete abortion & & $12(25.00 \%)$ \\
\hline MROP & & $8(16.67 \%)$ \\
\hline
\end{tabular}

Table 4: MTP related Complications $(\mathrm{n}=48)$

\begin{tabular}{|l|l|l|}
\hline & No. & $\%$ \\
\hline Need for D\&C & 4 & $8.33 \%$ \\
\hline MROP & 8 & $16.666 \%$ \\
\hline Need for BT & 2 & $4.166 \%$ \\
\hline Rupture / Hysterectomy & 0 & $0 \%$ \\
\hline Diarrhoea & 0 & $0 \%$ \\
\hline Fever & 5 & $1.041 \%$ \\
\hline Requirement for analgesics & & \\
None & 28 & $58.33 \%$ \\
Injectables & 18 & $37.5 \%$ \\
Oral & 2 & $4.17 \%$ \\
\hline Hospital Stay & $3.40(3.05-3.74)$ & $2-7$ days \\
\hline
\end{tabular}

\section{Discussion}

Mid-trimester MTP is a difficult situation owing to the prolonged time required for the abortion process and associated complications. In developing countries especially rural areas second trimester MTP is a real challenge owing to the limited resources available . mid-trimester pregnancy termination consisting prostaglandins alone or in combination with anti-progesterone Mifepristone. Still a method which is $100 \%$ reliable, safe and affordable is not known. There are many studies with different dosage schedules of combination of mifepristone and Misoprostol for $2^{\text {nd }}$ trimester MTP. We compared our study with other studies using a similar drug protocol of $200 \mathrm{mg}$ mifepristone followed by vaginal Misoprostol with minor variations of subsequent dosages of Misoprostol.

Various studies have shown higher success rate and reduced induction to abortion interval and need for lesser dose of misoprostol when mifepristone is added to misoprostol. $(3,8,9)$ Treatment with mifepristone softenes the cervix, increases sensitivity of uterus to prostaglandins. The maximum effect on uterine contractility and cervical ripening is seen after 36-48 hours. Three-fourth of our patients had complete expulsion of fetus and placenta within $48 \mathrm{hrs}, 25 \%$ required either dilatation \& curettage for retained products or manual removal of placenta. Different studies have shown success rates varying from $73 \%-97 \%$ with combination of mifepristone followed by vaginal misoprostol $(3,4,9)$.

We have given misoprostol vaginally $48 \mathrm{hrs}$ after priming with mifepristone. First dose administered was $400 \mathrm{mcg}$ followed by 100-200 mcg vaginally after 4-6 hrs depending upon uterine contractions and previous history of uterine surgery. It has been seen that vaginal route of administration for misoprostol is safer and more effective than oral route with less side-effects $(9,10)$ due to better bioavailability of the drug at target site. (11)

The mean total dose of misoprostol required in the present study was $600 \mathrm{mcg}(0-2400 \mathrm{mcg})$. Other studies' reported in literature show dosage requirements varying up to $2200 \mathrm{mcg}$. $(12,13)$ Mean induction to delivery interval was 11.26 hours which is comparable to other studies. $(5,9,10)$

Six of our patients (12.5\%) aborted completely with mifepristone alone and thus did not require misoprostol at all. Amongst these five patients (83.33\%) were diagnosed with intrauterine fetal demise. In study by Aggarwal $\mathrm{N}$ et al (10), $2.5 \%$ of patients aborted with mifepristone alone whereas other studies have reported $0.2 \%-0.5 \%$ incidence of complete abortion with only mifepristone. $(5,8,14)$

There were seven patients in our study who had undergone caesarean section prior to present pregnancy. All of them had complete abortion with combination of mifepristone and Misoprostol regimen. There are various case reports showing uterine rupture in previously scarred uterus undergoing mid-trimester pregnancy termination $(15,16)$ But, Many studies have shown safety of mifepristone \& misoprostol for mid-trimester MTP in cases of previous caesarean section. $(17,18)$

There was no case of rupture uterus or need for hysterotomy. Other minor complications included fever, need for D \&C. Majority of our cases didn't require any analgesics. Only 18 women required injectable analgesics 
and majority of these were nulliparous (55.56\%). Literature also shows similar finding with nulliparous women requiring more analgesics $(4,10)$ which can be due to their low threshold for pain.

\section{Conclusion}

Use of mifepristone with Misoprostol is effective and safe option for mid-pregnancy medical termination of pregnancy. In majority of cases surgical evacuation \& its complications can be avoided by using medical methods. The combination can be used in cases of previous scarred uterus under strict monitoring.Overall there was shortened IA interval, patient discomfort. Lower cost and hospital stay.Can be used successfully.

\section{References}

[1]. Medical Termination of Pregnancy Act, 1971.

[2]. World Health Organization (1997) Medical Methods for Termination of Pregnancy. WHO Technical Report Series 871. World Health Organization, Geneva

[3]. Lalitkumar S, Bygdeman M, Gemzell-Danielsson K. Mid-trimester induced abortion: a review. Hum Reprod Update 2007;13(1):37-52

[4]. Hammond C. Recent advances in second-trimester abortion: an evidence-based review. Am J Obstet Gynecol 2009;200(4):347-56

[5]. Ashok PW, Templeton A, Wagaarachchi PT, Flett GM. Midtrimester medical termination of pregnancy: a review of 1002 consecutive cases. Contraception 2004;69(1):51-8

[6]. Bygdeman M and Swahn ML. Progesterone receptor blockage. Effect on uterine contractility and early pregnancy. Contraception 1985;32, 4551.

[7]. Nagaria T, Sirmor N. Misoprostol vs mifepristone and Misoprostol in second trimester termination of pregnancy. J Obstet Gynaecol India 2011;61(6):659-62.

[8]. Hamoda H, Ashok PW, Flett GM, Templeton A. A randomized trial of mifepristone in combination with Misoprostol administered sublingually or vaginally for medical abortion at 13-20 weeks gestation. Hum Reprod 2005;20(8):2348-54.

[9]. Ho PC, Nagi SW, Liu KL, Wong GC and Lee Sw. Vaginal Misoprostol compared with oral Misoprostol in termination of second trimester pregnancy. Obstet Gynecol 1997;90:735-38.

[10]. Ngai SW, Tang OS and Ho PC. Randomised comparison of vaginal (200 meg every 3 hourly) and oral (400 mcg every 3 hourly) Misoprostol when combined with mifepristone in termination of second trimester pregnancy. Hum Rep 200;15:2205-8.

[11]. Tang OS, Chan CC, Kan AS and Ho PC. A prospective randomised comparison of sub-lingual and oral Misoprostol when combined with mifepristone for medical abortion at 12-20 weeks gestation. Hum Rep 2005;20:3062-66.

[12]. El- Refaey H and Templeton A. Induction of abortion in the second trimester by a combination of Misoprostol and mifepristone: a randomized comparison between two Misoprostol regimens. Hum Reprod. 1995;10:475-78.

[13]. Agarwal N, Gandhi G, Batra S, Sharma R. Evaluation of mifepristone and Misoprostol for medical termination of pregnancy between 13-20 weeks of gestation. Indian Journal of Clinical practice. 2014;24(9):859-62.

[14]. Gupta N, Mittal S. Is mifepristone needed for second trimester termination of pregnancy?JTurkish-German Gynecol Assoc 2007;8(1):58-62.

[15]. Chen M, Shih Jc, Chiu WT, Hsieh FJ. Separation of caesarean scar during second trimester intravaginal Misoprostol abortion. Obstet Gynaecol 1999;94:840.

[16]. Berghahn L Christensen D and Droste S. Uterine rupture during second trimester abortion associated with Misoprostol. Obstet Gynaecol 2001;98:976-77.

[17]. Dickinson JE. Misoprostol for second trimester pregnancy termination in women with prior caesarean delivery. Obstet Gynaecol 2005;105:352-56.

[18]. Herabutya Y, Chanarachakul B and Punyavachira P. Induction of labour with vaginal Misoprostol for second trimester termination of pregnancy in the scarred uterus. Int J Gynaecol Obstet 2003;83:293-97. 